\title{
Choosing a Global Architecture for Mobile Applications
}

\author{
Robin Nunkesser \\ Hamm-Lippstadt University of Applied Sciences \\ Email: robin.nunkesser@hshl.de
}

\begin{abstract}
Complex mobile applications require an appropriate global architecture. If used correctly, the high-level design patterns promoted by Apple and Google such as MVC, MVVM, and MVI/MVU may make an important contribution to the architecture, but they often require supplementary architectural concepts. General architectures such as Clean Architecture may come to the rescue but leave room for interpretation as to how they work best on iOS and Android. This paper gives an overview of the current situation and choices and a vision of using a mix-and-match approach with the following goals: provide an extendable approach suitable for small and large projects, use minimal integration into the platform, and sidestep traps and erroneous implementations.
\end{abstract}

\section{INTRODUCTION}

When Apple published the iPhone SDK in 2008, they recommended adhering to the guidelines of the Cocoa Fundamentals Guide. A central role is played by their flavor of the Model View Controller (MVC) pattern originally introduced by Smalltalk (see e.g. [1]), which is described in [2] as a "high-level pattern in that it concerns itself with the global architecture of an application". Technically, it is mainly a combination of GoF patterns from [3] and a categorisation of objects into the three categories of models, views, and controllers (see Figure 1).

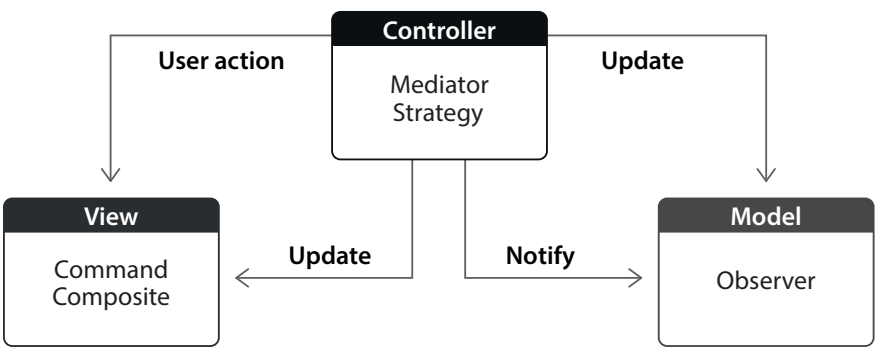

Figure 1. Reproduction of Apple MVC from [2]

The first templates for the iPhone SDK provided an UIView as a view class, an Application Delegate as a controller class, and no example model classes. The first Android templates were similar; they had an XML layout file providing a view class and an Activity as a controller class. The missing model examples in these templates contributed to a problem from which many apps suffer: too much code in controller classes.

Apple, Google, and mobile frameworks such as Xamarin and Flutter have introduced or are planning to introduce variations of Model View Controller inspired by Model-ViewIntent (MVI; introduced in [4]) and Model View Update
(MVU; introduced in [5]) in 2021 as a new recommended variation of MVC (with SwiftUI, Jetpack Compose, .NET Multi-platform App UI, for example).

The corresponding templates typically feature a declarative UI example for the view and a State example exemplifying the model (State is a concept to describe model changes that affect the presentation). Updating is implicitly handled by the frameworks. Again, these templates lack good model examples for data and logic not directly coupled to the presentation.

As stated above, MVC, MVI, MVU, and all the variations used and discussed in between, such as Model View Presenter (MVP) and Model/View/ViewModel (MVVM) (e.g. in Xamarin and Android Jetpack ViewModel) are high-level patterns contributing to the global architecture, but they are clearly not global architectures which suffice for complex mobile applications.

Looking deeper into the architectural choices that must be made for mobile applications is therefore of great interest.

\section{RELATED WORK}

There are many articles in blogs and non-reviewed digital publications on architectural topics for mobile applications, but the focus here is on scientific publications.

Many publications such as [6], [7], [8] are concerned with special cases and not global solutions. Publications such as [9], [10], [11] focus on high-level patterns and not on global architectures. Sommerville [12] mostly treats mobile applications and web applications equally and only seldom expands on the special requirements of mobile apps.

Dobrean and Dioşan [13] present a comparative study of MVC, MVP, MVVM, and VIPER (an architecture proposed for iOS in [14]).

Salazar and Brambilla [15] concentrate on a high-level process to guide application developers in the task of designing a suitable software architecture.

\section{REQUIREMENTS OF MOBILE APP ENGINEERING}

Mobile application engineering has some specific aspects that differ from traditional software engineering:

- A gap between development and runtime hardware

- A high amount of UI code

- Short release cycles of hardware and software

- A large amount and variety of sensors and actors

- Customisation for individual device types

- Data storage, synchronisation, and offline availability 
- Event and lifecycle-based programming

- Concurrency with a special role for the UI thread

- Need for efficient code (memory, power, ...)

This list is not meant to be exhaustive. It focusses on aspects that are relevant for architectural choices. The works of Wasserman [16], König-Ries [17], Vollmer [18], Knott [19], and Sommerville [20], [12] may be of interest for details and further aspects.

Two architectural goals are especially helpful in coping with these aspects: independence of frameworks and external agencies and testability.

1) Independence: Independence of frameworks and external agencies is essential for mobile applications. It can help to cope with the gap between development and runtime hardware by enabling code components to run on both hardwares, helping with unit tests and reuse beyond iOS and Android. By offering better flexibility, it also helps with the short release cycles, large variety of hardware and software, and customisation needs.

2) Automatic Testing: Many of the stated aspects of mobile applications contribute to the complexity of testing for mobile applications. While the benefits of automatic testing are well known (see e.g. [21]), we also know e.g. from Beller, Gousios, Panichella, and Zaidman [22], for example, that many developers do not test. This is especially true for the mobile world, where we should divide automatic tests into at least three classes: local unit tests (on the development hardware), unit tests (on the runtime hardware), and UI tests. One of the main benefits of unit tests given by Kent in [23] is rapid feedback, which is only achieved by local unit tests. Therefore, good global architectures support local unit tests.

\section{THE PRESENT}

Currently, developers and architects typically either use the endemic (see e.g. [24]) high-level patterns such as MVC/MVP/MVVM/MVI/MVU (abbreviated MVX from now on), third-party patterns such as Functional Reactive Programming, or a mobile adaption of a global architecture such as Martin's Clean Architecture from e.g. [25]. In the following, we will focus on MVX and Clean Architecture. The reason to omit Functional Reactive Programming here is that it typically tightly integrates external dependencies which in itself prohibits to achieve the desired independence of frameworks and external agencies.

\section{A. $M V X$}

An oft-stated criticism of MVC, for example, is that it leads to large controller classes (see e.g. [8]). While the typical reason for this is more likely to be the incorrect application of the pattern by developers, Apple and Google have contributed to these problems by providing templates without model examples, for example. As Salazar and Brambilla state in [15]: many developers follow "the false intuition that [...] following the guidelines of platform developers is enough to guarantee that a mobile application would have a good architectural design." In a way, iOS and Android also suffered from their

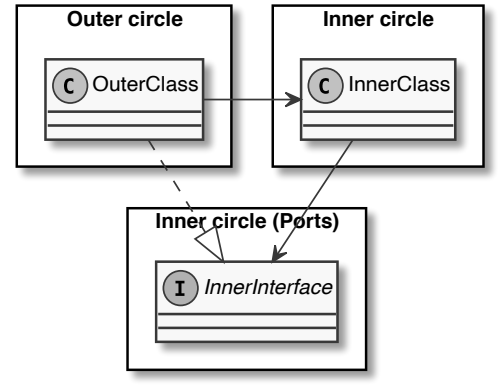

Figure 2. Boundary Crossing

success: the ratio of inexperienced developers in comparison to experienced developers was very high in the beginning.

Modern smartphones have a lot of computing power and developers have the freedom to execute complex tasks on the device or in the cloud. For apps with a lot of business logic, view-focused MVX patterns are not enough. Even apps that make heavy use of cloud services may be in need of an architecture that allows flexibility in changing these services and testability for the external dependencies.

However, MVX may suffice if it is done right and the mobile application is mostly concerned with presentation tasks.

\section{B. Modern Global Architectures}

VIPER [14], VIP [26], and CleanArchitectureRxSwift ${ }^{1}$ attracted a lot of attention on iOS. Android-CleanArchitecture ${ }^{2}$ (see also [27]), Wojda's Android Showcase ${ }^{3}$, and Kušt's Clean Architecture Tutorial [28] are popular examples on Android. All of them are an application of Martin's Clean Architecture [29] to mobile apps.

Clean Architecture integrates ideas from preceding architectural concepts such as Ports and Adapters (a.k.a. Hexagonal Architecture) by Cockburn [30], Onion Architecture by Palermo [31], DCI from Coplien and Reenskaug [32] and EIC/EBI by Jacobson [33], and adds principles and concepts.

Clean Architecture proposes using four onion-style layers (also called circles; see e.g. [31]): Entities, Use Cases, Interface Adapters, and Frameworks and Drivers. Its main objectives according to [29] are appropriately independence of frameworks and external agencies (UI, Databases, ...) and testability. As stated above, these objectives are helpful for all kinds of software, but especially helpful for mobile applications. A major part of achieving this is using the dependency inversion principle to cross circle boundaries. Inner circle classes may only communicate with outer circle classes through interfaces implemented by outer circle classes (see Figure 2 for an example).

One major problem posed by Clean Architecture is the lack of official reference implementations for different platforms (in

\footnotetext{
${ }^{1}$ https://github.com/sergdort/CleanArchitectureRxSwift

${ }^{2}$ https://github.com/android10/Android-CleanArchitecture-Kotlin

${ }^{3}$ https://github.com/igorwojda/android-showcase
} 


\begin{tabular}{l|cccc} 
& Independence & Testability & Modularity & Faithfulness \\
\hline VIPER & high & medium & low & medium \\
VIP & high & high & low & high \\
CA-RxSwift & low & medium & high & medium \\
Android-CA & medium & medium & medium & high \\
Wojda & medium & high & high & high \\
Kušt & high & high & high & medium \\
& \multicolumn{4}{c}{ Table I }
\end{tabular}

COMPARISON OF EXISTING CleAN ARCHITECTURE IMPLEMENTATIONS

Martin's books, his blog, his training videos, and his GitHub account, for example). The official examples are FitNesse ${ }^{4}$ (a real world project not tailored to learning Clean Architecture), Payroll (e.g. in [34] made with similar principles but not the same naming conventions as Clean Architeture), Video Sales (in [29] which is surprisingly brief), and the CleanCodeCaseStudy ${ }^{5}$ which is well suited to learning Clean Architecture but not promoted as a Clean Architecture example. There is even an example for $\mathrm{iOS}^{6}$, which is very different from VIP and VIPER, but also misses some useful ideas presented in [29].

The suboptimal situation with regard to official reference implementations leads to at least two problems: a large variety of unofficial examples (In January 2021 a search for "Clean Architecture" on GitHub returns 626 repositories with Swift examples and even 1.915 repositories with Kotlin examples) with little quality assurance and often conflicting concepts. Table I provides an evaluation of the six approaches mentioned with regard to the following criteria:

1) Independence: How independent is the approach of external agencies?

2) Testability: How amenable is the approach to unit tests, and especially local unit tests?

3) Modularity: Is modularity supported and encouraged?

4) Faithfulness: How much does it deviate from Clean Architecture ideas?

Of the examples considered, none matches all expectations fully. As an example, we take a look at the essential boundary crossing concept from Figure 2. This is violated by VIPER, CA-RxSwift, and Kušt resulting in lower grades in faithfulness.

\section{THE VISION}

A common problem in the application of MVX and Clean Architecture is that a suboptimal starting point (incomplete templates, missing reference implementations) has led to a variety of embodiments with sometimes conflicting and sometimes even incorrect interpretations. As a consequence, Clean Architecture examples for iOS and Android tend to give a lot of platform-specific guidance often sacrificing independence and sustainability.

It is useful to reiterate that MVX are high-level patterns incorporating design patterns and a taxonomy. Clean Architecture, on the other hand, also incorporates a taxonomy, design

\footnotetext{
${ }^{4}$ https://github.com/unclebob/fitnesse

${ }^{5}$ https://github.com/cleancoders/CleanCodeCaseStudy

${ }^{6}$ https://github.com/unclebob/MACS_GOMOKU
}

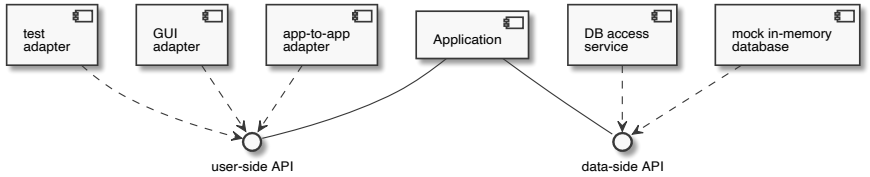

Figure 3. Ports and Adapters (Reproduction from [30])

principles, component principles, a layering concept, and a boundary concept, among other ideas. A mobile application project with a good global architecture should not start with an unofficial template already containing a number of platform dependent frameworks; it should use all concepts that are useful for mobile development in a way that stays loosely coupled to the platform. So let us take a look at some of the influences and building blocks of modern architectures and why they may be helpful for mobile development. On this basis, a mix-andmatch approach may be used that is extendable for small and large projects and preserves independence.

\section{A. Design Patterns}

When talking about high-level patterns and architectural concepts, we should not forget design patterns such as the GoF-Patterns that may, of course, also be used and integrated independently.

\section{B. SOLID Design Principles}

Clean architecture promotes the well known SOLID design principles (see e.g. [34]). As they contribute to the general architectural goals of Clean Architecture, they are naturally also relevant for mobile applications.

\section{Entity-Interface-Control / Entity-Boundary-Interactor}

Jacobson [33] proposes structuring systems with three object types. The Entity-Interface-Control types originally chosen where later changed to Entity-Boundary-Control and then Entity-Boundary-Interactor. Entity objects hold the data used by the system and all the behaviour naturally coupled to this data. Boundary objects model the interface with the system. All the behaviour that remains is placed in Interactor objects. Martin's Clean Architecture adapts these object types. The boundary objects in particular help with decoupling and therefore independence and testability. However, EBI is not very explicit in how to gain independence from more than just the UI.

\section{Ports and Adapters / Hexagonal Architecture}

Cockburn introduces Ports and Adapters (formerly known as Hexagonal Architecture) in [30] and explicitly states the aim to: "Allow an application to equally be driven by users, programs, automated test or batch scripts, and to be developed and tested in isolation from its eventual run-time devices and databases." The idea incorporates an application core with ports (protocols or interfaces) that define how the application may be used (by so called driving adapters implemented in the core) and what data the application needs (provided by so 


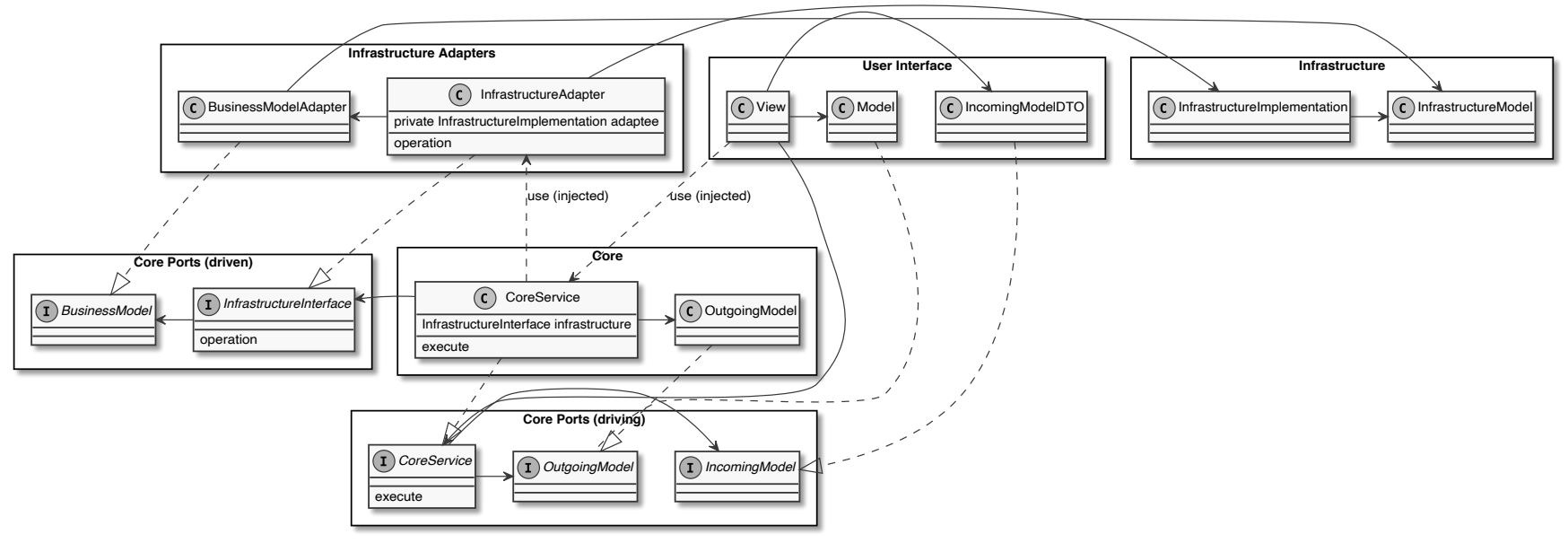

Figure 4. Minimal Example

called driven adapters implemented externally) (see Figure 3 for an illustration).

Ports and Adapters has a very easy concept for achieving the main architectural goals discussed here. However, the biggest advantage is that it may be easily combined with known concepts. Vernon states in [35]: "Because the Hexagonal Architecture is versatile, it could well be the foundation that supports other architectures required by the system. [...] The Hexagonal style forms the strong foundation for supporting any and all of those additional architectural options."

Ports and Adapters with MVVM for the GUI and EBI for the application core, for example, is a valid implementation of Clean Architecture. Using DDD [36] for the application core provides the basis for Palermo's Onion Architecture [31] and Graça's Explicit Architecture [37]. Building your architecture upon this idea also facilitates using MVI/MVU.

\section{E. Components / Packaging / Modularisation}

Brown [38] discusses a lot of different methods of packaging the code. He also discusses the question of enforcement and creating separate assemblies for the components. While iOS and Android do not include modularisation in the default templates, it is fairly easy to modularise the code and this also holds true for many cross-platform frameworks. Separating the code into real modules in mobile applications has a lot of advantages: testability, possible division of platform dependent and platform independent code, flexibility and, of course, easier enforcement of architectural principles. Brown recommends a "package by component" approach which deviates a little from the Clean Architecture ideas. This is a good example on why it is helpful to refrain from seeing Clean Architecture as a monolithic concept.

\section{F. Dependency Injection}

Dependency injection helps with keeping the components independent and with testability. This does not necessarily mean that a dependency injection framework is needed, however. It may be enough to consider which dependencies have to be flexible and organise them in a transparent way that is easy to change.

\section{G. Minimal Example}

Figure 4 provides a minimal example using most of the concepts mentioned. It is important to note, that this is not an alternative proposal to Clean, Onion, or Explicit Architecture. It is intended as a foundation or common ground which can be built upon. In a way it is a concretion of Ports and Adapters that is in full accordance with Clean, Onion, and Explicit Architecture.

As mentioned before, for simple apps with not much business logic, MVX may suffice. There are also apps of medium complexity that do not need external infrastructure or do not need separate models for the layers. As the layers are strictly separated in Figure 4, modifications for these situations are straightforward. With these considerations in mind, the example is minimal with regard to all concepts a complex application may need, but it may be adopted and further minimised on demand.

Mobile applications are event and lifecycle-based (see e.g. [12]) and a typical control flow starts, for example, with a tap in a view. The User Interface should be free of business logic, which should be provided by the application core (and the infrastructure). The view may trigger actions in the application core with an interface whose concrete implementation (typically called Interactor, Service, or Command) gets injected. The view may provide data through a data transfer object and has different options for receiving notification of the results. The concrete implementation should depend on the MVX flavor used. Sometimes, the application core requires external infrastructure to fulfill the business logic. The infrastructure needed is described in an interface (potentially also with additional model interfaces). The adapter pattern is used to provide the option of using third-party infrastructure implementations and flexibly changing the implementation. The concrete adapter is also injected. 


\section{CONCLUSION}

Popular modern architectural approaches such as Clean, Onion, and Explicit Architecture are essential for complex mobile applications because they assure independence of external frameworks and testability. However, the lack of qualityassured reference implementations for iOS and Android lead to a variety of implementations and examples of varying quality. One reason for this is that Clean Architecture is often seen as a lock, stock, and barrel approach and is also frequently integrated too tightly with platform-specific or even external techniques. It is therefore helpful to look at the building blocks of such architectures and to obtain a toolset to tailor your own architectural approach.

\section{REFERENCES}

[1] T. Reenskaug, "Models - views - controllers," Xerox PARC, Tech. Rep., 1979.

[2] Apple Computer, Inc., "Cocoa fundamentals guide," 2006.

[3] E. Gamma, R. Helm, R. Johnson, and J. Vlissides, Design Patterns. Elements of Reusable Object-Oriented Software. Boston, MA: AddisonWesley, 1995.

[4] A. Staltz, "Model-view-intent," Cycle.js, 2015. [Online]. Available: https://cycle.js.org/model-view-intent.html

[5] E. Czaplicki, "The elm architecture," guide.elm-lang, 2016. [Online]. Available: https://guide.elm-lang.org/architecture/

[6] H. J. La and S. D. Kim, "Balanced mvc architecture for developing service-based mobile applications," in 2010 IEEE 7th International Conference on E-Business Engineering, 2010, pp. 292-299.

[7] D. Wichmann, M. Pielot, and S. Boll, "Companion platform - modulare softwareplattform zur schnellen entwicklung von mobilen anwendungen," it - Information Technology, vol. 51, no. 2, pp. 72 - 78, 2009.

[8] M. Aljamea and M. Alkandari, "Mmvmi: A validation model for mvc and mvvm design patterns in ios applications," IAENG International Journal of Computer Science, vol. 45, no. 3, pp. 377-389, 2018.

[9] D. Plakalovic and D. Simic, "Applying mvc and pac patterns in mobile applications," ArXiv, vol. abs/1001.3489, 2010.

[10] F. E. Shahbudin and F. Chua, "Design patterns for developing high efficiency mobile application," Journal of Information Technology \& Software Engineering, vol. 3, pp. 1-9, 2013.

[11] K. Sokolova and M. Lemercier, "Towards high quality mobile applications: Android passive mvc architecture," International Journal On Advances in Software 1942-2628, vol. 7, pp. 123 - 138, 2014.

[12] I. Sommerville, Engineering Software Products. London: Pearson, 2020.

[13] D. Dobrean and L. Dioșan, "A comparative study of software architectures in mobile applications," Studia Universitatis Babeș-Bolyai Informatica, vol. 64, no. 2, pp. 49-64, 2019.

[14] J. Gilbert and C. Stoll, "Architecting ios apps with viper," objc, vol. 13, 2014. [Online]. Available: https://www.objc.io/issues/13architecture/viper/

[15] F. J. A. Salazar and M. Brambilla, "Tailoring software architecture concepts and process for mobile application development," in Proceedings of the 3rd International Workshop on Software Development Lifecycle for Mobile, ser. DeMobile 2015. New York, NY, USA: Association for Computing Machinery, 2015, p. 21-24.

[16] A. Wasserman, "Software engineering issues for mobile application development," 2010, pp. 397-400.

[17] B. König-Ries, "Challenges in mobile application development," it Inf. Technol., vol. 51, no. 2, pp. 69-71, 2009.

[18] G. Vollmer, Mobile App Engineering: Von den Requirements zum Go Live. Heidelberg: dpunkt.verlag, 2017.

[19] D. Knott, Hands-On Mobile App Testing. Boston, MA: Addison-Wesley Professional, 2015.

[20] I. Sommerville, Software Engineering, 10th ed. London: Pearson, 2016.

[21] D. M. Rafi, K. R. K. Moses, K. Petersen, and M. V. Mäntylä, "Benefits and limitations of automated software testing: Systematic literature review and practitioner survey," in 2012 7th International Workshop on Automation of Software Test (AST), 2012, pp. 36-42.
[22] M. Beller, G. Gousios, A. Panichella, and A. Zaidman, "When, how, and why developers (do not) test in their ides," in Proceedings of the 2015 10th Joint Meeting on Foundations of Software Engineering, ser. ESEC/FSE 2015. New York, NY, USA: Association for Computing Machinery, 2015, p. 179-190.

[23] K. Beck, Test Driven Development: By Example. Boston, MA: AddisonWesley Professional, 2002.

[24] R. Nunkesser, "Beyond web/native/hybrid: A new taxonomy for mobile app development," in Proceedings of the 5th International Conference on Mobile Software Engineering and Systems, ser. MOBILESoft '18. Piscataway, NJ: IEEE Press, 2018.

[25] R. C. Martin. (2012) The clean architecture. [Online]. Available: https://8thlight.com/blog/assets/posts/2012-08-13-the-clean-architecture

[26] R. Law, The Clean Swift Handbook, 2019. [Online]. Available: https://clean-swift.com/handbook/

[27] F. Cejas, "Architecting android...reloaded." fernandocejas.com, 2019 [Online]. Available: https://fernandocejas.com/blog/engineering/201905-08-architecting-android-reloaded/

[28] I. Kušt, "Clean architecture tutorial for android: Getting started," raywenderlich.com, 2019. [Online]. Available: https://www.raywenderlich.com/3595916-clean-architecturetutorial-for-android-getting-started

[29] R. C. Martin, Clean Architecture - A Craftsman's Guide to Software Structure and Design. Englewood Cliffs, NJ: Prentice Hall, 2017.

[30] A. Cockburn, "Hexagonal architecture," alistair.cockburn.us, 2005. [Online]. Available: http://alistair.cockburn.us/Hexagonal+architecture

[31] J. Palermo, "Onion architecture," jeffreypalermo.com, 2008. [Online]. Available: https://jeffreypalermo.com/2008/07/the-onion-architecturepart-1/

[32] J. Coplien and G. Bjørnvig, Lean Architecture: for Agile Software Development. Wiley, 2011.

[33] I. Jacobson, M. Christerson, P. Jonsson, and G. Övergaard, ObjectOriented Software Engineering. New York, NY, USA: ACM, 1992.

[34] R. Martin and M. Martin, Agile Principles, Patterns, and Practices in C\#, ser. Robert C. Martin Series. Pearson Education, 2006.

[35] V. Vernon, Implementing Domain-Driven Design. Addison-Wesley Professional, 2013.

[36] E. Evans, Domain-Driven Design. Boston, MA: Addison-Wesley Professional, 2003.

[37] H. Graça, "Ddd, hexagonal, onion, clean, cqrs, ... how i put it all together," herbertograca.com, 2017. [Online]. Available: https://herbertograca.com/2017/11/16/explicit-architecture01-ddd-hexagonal-onion-clean-cqrs-how-i-put-it-all-together/

[38] S. Brown, "The missing chapter,' in Clean Architecture - A Craftsman's Guide to Software Structure and Design, R. C. Martin, Ed. Englewood Cliffs, NJ: Prentice Hall, 2017, pp. 303-321. 